\title{
Die Umsetzung der FFH-Richtlinie in der Agrarlandschaft
}

\author{
Eckhard Schröder • Axel Ssymank • Mareike Vischer-Leopold $\cdot$ Marion Ersfeld
}

Erhalten: 18. Februar 2008/Akzeptiert: 20. August 2008/Online veröffentlicht: 13. September 2008

(C) Springer-Verlag 2008

Zusammenfassung Die Fauna-Flora-Habitat-Richtlinie (FFH-RL, 92/43/EWG) enthält neben dem Aufbau des Netzes Natura 2000 mit Arten und Lebensraumtypen auch Regeln für den Artenschutz. Dreiviertel aller gemeldeten FFH-Gebiete beinhalten neben naturnahen Biotoptypen wie Wäldern und Gewässern auch landwirtschaftliche Flächen. Wobei $18 \%$ der Gebiete landwirtschaftliche Nutzflächen mit einem Anteil zwischen 25 und $50 \%$ enthalten und $24 \%$ sogar einen Anteil von über 50\%. 48 Arten und drei Lebensraumtypen der FFH-RL haben einen Schwerpunkt im Agrarbereich. Hinzu kommen elf Lebensraumtypen, die von einer naturschutzgerechten landwirtschaftlichen Nutzung abhängig sind. Ein großer Teil dieser Lebensräume und Arten befindet sich derzeit nicht in einem günstigen Erhaltungszustand. Der folgende Beitrag analysiert zum einen das Betroffensein der Landwirtschaft durch die Lebensraumtypen und Arten der FFH-RL, zum anderen werden einige indirekte negative Wirkungen aufgezeigt, die erheblichen Einfluss auf die Entwicklung der Erhaltungszustände von Lebensräumen und Arten haben können.

Schlüsselwörter Agrarlandschaft · Biodiversität . FFH-Richtlinie $\cdot$ Natura $2000 \cdot$ Naturschutz

Herausgeber: Martina Roß-Nickoll · Christoph Schulte

E. Schröder $(\bowtie) \cdot$ A. Ssymank $\cdot$ M. Vischer-Leopold $\cdot$ M. Ersfeld Bundesamt für Naturschutz

Konstantinstr. 110, 53179 Bonn, Deutschland

E-Mail: SchroedE@BfN.de
The implementation of the EU Habitats Directive in agricultural landscapes

\begin{abstract}
The EU Habitats Directive (92/43/EEC) does include provisions for setting up the Natura 2000-network of protected areas based on listed species and habitats, and in addition specific regulations on species protection. Three Quarters of all designated sites (SCI's) do not only include natural habitat types in a strict sense like forests or waterbodies, but also agricultural land. $18 \%$ of the SCI's even include between 25 and $50 \%$ agricultural land and $24 \%$ above $50 \%$. 48 species and three habitat types listed under the Habitats Directive have a clear focus in agriculture. Another eleven habitat types are dependant from a nature-friendly low intensity use or management. A large proportion of these habitats and species are actually in an unfavourable conservation status. The paper analyses the impact of EU nature conservation on agriculture based on the species and habitats falling under the Habitats Directive. On the other hand indirect negative influences of agriculture are discussed, that may have considerable impact on the future development of the conservation status of endangered habitats and species.
\end{abstract}

Keywords Agricultural landscape $\cdot$ Biodiversity $\cdot$ Habitats Directive $\cdot$ Natura $2000 \cdot$ Nature conservation

\section{Einführung}

Die Einführung der „Fauna-Flora-Habitat-Richtlinie“ (FFHRL) (Der Rat der Europäischen Gemeinschaften 1992) legte den Grundstein für einen umfassenden europaweiten Lebensraum- und Artenschutz. Die wesentlichen Ziele der FFH-RL können mit dem Erhalt der biologischen Vielfalt auf der europäischen Ebene zusammengefasst werden. Zur 
Abb. 1 In der EU werden derzeit neun biogeografische Regionen unterschieden, von denen drei auch in Deutschland vorkommen

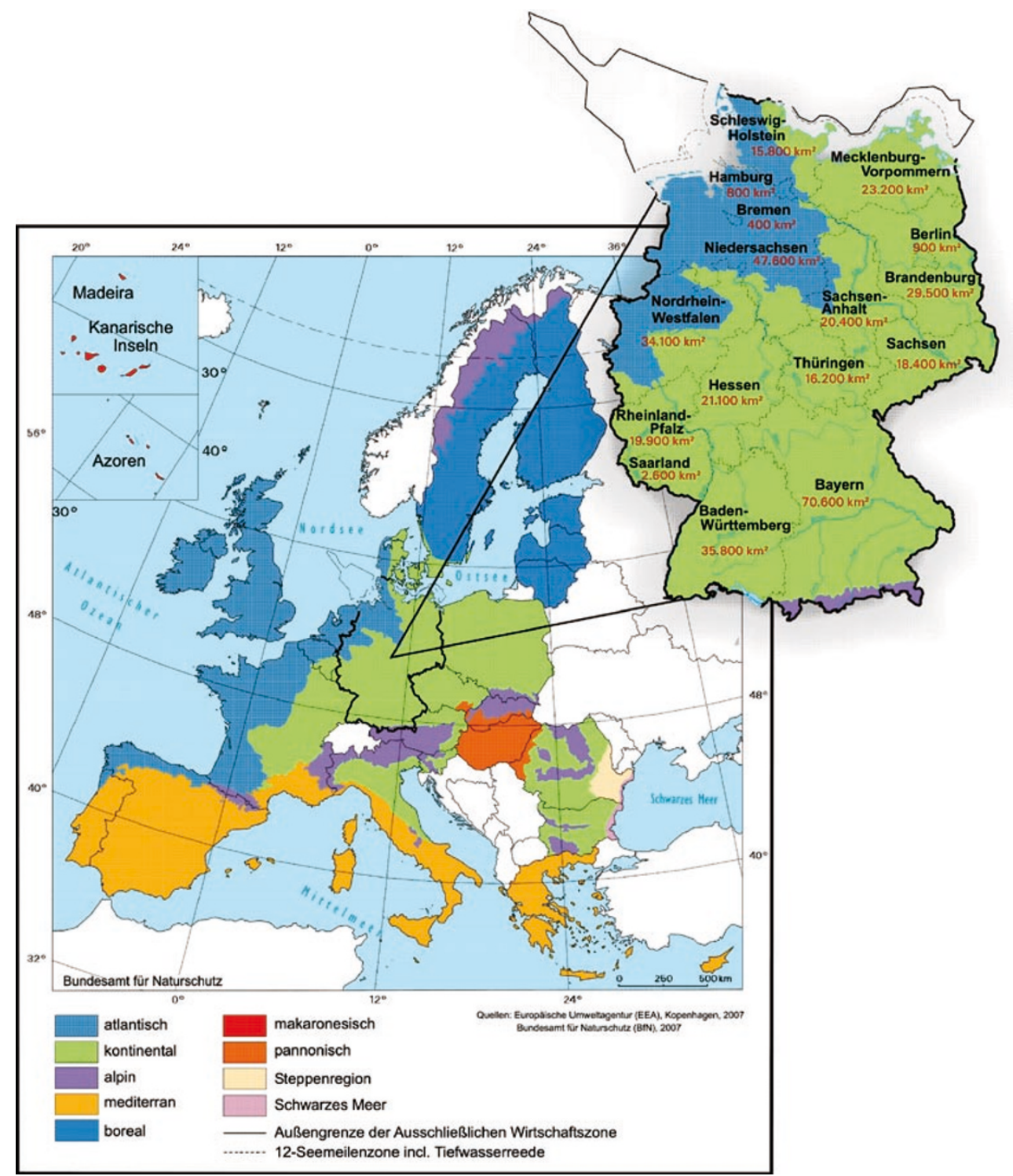

Richtlinie gehören insgesamt sechs Anhänge, von denen im Anhang I die Lebensraumtypen (LRT) und im Anhang II die Arten erfasst sind (Ssymank et al. 1998), für die die Mitgliedstaaten der EU-Schutzgebiete auszuwählen hatten. Die Arten des Anhang IV unterliegen dagegen einem strengen Artenschutz - auch außerhalb der Schutzgebiete - und der Anhang V regelt den Artenschutz für wirtschaftlich nutzbare Arten. Über das Betroffensein der Landwirtschaft durch den europäischen Naturschutz herrschen erhebliche Unsicherheiten, obwohl die Flächenanteile landwirtschaftlicher Flächen in den FFH-Gebieten wesentlich geringer sind als die Waldanteile. Der nachfolgende Artikel soll hier durch eine systematische Analyse der betroffenen Lebensraumtypen und Arten und vor dem Hintergrund der vorliegenden Bewertungen zum Erhaltungszustand aus dem nationalen Bericht 2007 (Art. 17 FFH-Richtlinie, Der Rat der Europä- ischen Gemeinschaften 1992) einen besseren Einblick verschaffen. Darüber hinaus werden indirekte Einwirkungen der Landwirtschaft auf das Netz Natura 2000 diskutiert.

\section{Welche Lebensraumtypen und Arten in Agraröko- systemen gibt es?}

In den Anhängen der FFH-RL werden für die 27 Mitgliedstaaten in den neun biogeografischen Regionen (Abb. 1) der EU 231 Lebensraumtypen (LRT) aufgeführt (Der Rat der Europäischen Union 2006), von denen 91 auch in Deutschland vorkommen. Unter diesen 91 LRT werden hier 20 LRT als mehr oder weniger bedeutsam für den Agrarbereich eingestuft (European Commission, DG Umwelt 2007). Es werden im Folgenden sowohl bei den Lebensraumtypen als auch bei 
Tabelle 1 LRT die „mittelintensiv“ agrarisch genutzt werden

\begin{tabular}{ll}
\hline Code & Lebensraumtyp der Kategorie I \\
\hline 6440 & Brenndolden-Auenwiesen \\
6510 & Magere Flachland-Mähwiesen \\
6520 & Berg-Mähwiesen \\
\hline
\end{tabular}

den Arten drei Kategorien (mittelintensive, extensive Agrarlandschaft, mögliche indirekte Beeinflussung) unterschieden. Bei den LRT der Kategorie I handelt es sich um Elemente der mittelintensiv genutzten Agrarlandschaft (Tabelle 1), während diejenigen LRT der Kategorie II durch extensive Landwirtschaft betroffen sind (Tabelle 2). Die LRT der noch verbleibenden Kategorie III werden dagegen nur indirekt von landwirtschaftlichen Maßnahmen beeinflusst. Hierunter fallen etwa an Agrarflächen direkt angrenzende LRT oder Habitate der Anhangsarten oder auch diejenigen, die durch landwirtschaftliche Emissionen beeinträchtigt werden können.

Von den 911 Arten des Anhanges II der EU-Mitgliedstaaten sind in Deutschland 133 vertreten. Für den Anhang IV kommen 132 von EU-weit insgesamt 1026 Arten hinzu, die sich aber z.T. mit dem Anhang II überschneiden. Es verbleiben dann noch 87 Arten (von 223 in der EU) des Anhanges V. Von den Arten der Anhänge der FFH-Richtlinie werden hier elf Arten der ,mittelintensiv“ genutzten Agrarlandschaft zugeordnet (Tabelle 3).

Zu den Arten der Kategorie II (Arten der extensiven Agrarlandschaft) gehören z. B. Arten, die ihren Habitatschwerpunkt im trockenen und feuchten mageren Grünland oder in Heiden besitzen wie Berg-Wohlverleih (Arnica montana), verschiedene Bärlapparten (Diphasiastrum spec., Huperzia selago, Lycopodium clavatum), Rentierflechten (Cladonia Subgen. Cladina), viele Fledermausarten, Moor- und Gras-
Tabelle 3 Arten der ,mittelintensiv“ genutzten Agrarlandschaft (Kategorie I)

\begin{tabular}{ll}
\hline Wissenschaftlicher Name & Deutscher Name \\
\hline Bromus grossus & Dicke Trespe \\
Notothylas orbicularis & Kugel-Hornmoos \\
Cricetus cricetus & Feldhamster \\
Myotis nattereri & Fransenfledermaus \\
Plecotus austriacus & Graues Langohr \\
Pelobates fuscus & Knoblauchkröte \\
Elaphe longissima & Äskulapnatter \\
Lacerta bilineata & Westliche Smargdeidechse \\
Podarcis muralis & Mauereidechse \\
Maculinea nausithous & Dunkler Wiesenknopf-Ameisen- \\
& bläuling \\
Maculinea teleius & Heller Wiesenknopf-Ameisen- \\
\end{tabular}

frosch (Rana arvalis, Rana temporaria), die Schlingnatter (Coronella austriaca) oder der Große Feuerfalter (Lycaena dispar). Insgesamt werden hier 37 Arten dieser Kategorie zugeordnet. In die Kategorie III gehören zusätzlich zu den terrestrischen Arten auch aquatische Arten wie Fische und Libellen, die indirekt z. B. durch landwirtschaftlich bedingte Stickstoffdepositionen betroffen sein können.

\section{Schutz der Lebensraumtypen}

Lebensraumtypen des Anhangs I der FFH-Richtlinie unterliegen in den gemeldeten FFH-Gebieten des europäischen Schutzgebietsnetzes Natura 2000 einem Schutzregime, welches ein Verschlechterungsverbot des Erhaltungszustandes (Art. 6 Abs. 2) und bei Eingriffen eine FFH-Verträglichkeitsprüfung

Tabelle 2 LRT die „,extensiv“ agrarisch genutzt werden

\begin{tabular}{|c|c|c|c|}
\hline Code & Lebensraumtypen der Kategorie II & Code & Lebensraumtypen der Kategorie II \\
\hline 1330 & Atlantische Salzwiesen & 6210 & $\begin{array}{l}\text { Kalk-(Halb-)Trockenrasen und ihre Verbuschungsstadien } \\
\text { ( }{ }^{\text {a }} \text { orchideenreiche Bestände) }\end{array}$ \\
\hline 2310 & $\begin{array}{l}\text { Sandheiden mit Besenheide und Ginster auf Binnendü- } \\
\text { nen }\end{array}$ & $6230^{\text {a }}$ & Artenreiche Borstgrasrasen \\
\hline 2320 & Sandheiden mit Krähenbeere auf Binnendünen & $6240^{\text {a }}$ & Steppenrasen \\
\hline 2330 & $\begin{array}{l}\text { Offene Grasflächen mit Silbergras und Straußgras auf } \\
\text { Binnendünen }\end{array}$ & 6410 & Pfeifengraswiesen \\
\hline 4010 & Feuchte Heiden mit Glockenheide & 6430 & Feuchte Hochstaudenfluren \\
\hline 4030 & Trockene Heiden & 7230 & Kalkreiche Niedermoore \\
\hline 4060 & Alpine und boreale Heiden & - & - \\
\hline 5130 & $\begin{array}{l}\text { Wacholderbestände auf Zwergstrauchheiden oder Kalk- } \\
\text { rasen }\end{array}$ & - & - \\
\hline $6110^{*}$ & Basenreiche oder Kalk-Pionierrasen & - & - \\
\hline $6120 *$ & Subkontinentale basenreiche Sandrasen & - & - \\
\hline 6170 & Alpine und subalpine Kalkrasen & - & - \\
\hline
\end{tabular}

${ }^{a}$ Prioritärer Lebensraum 


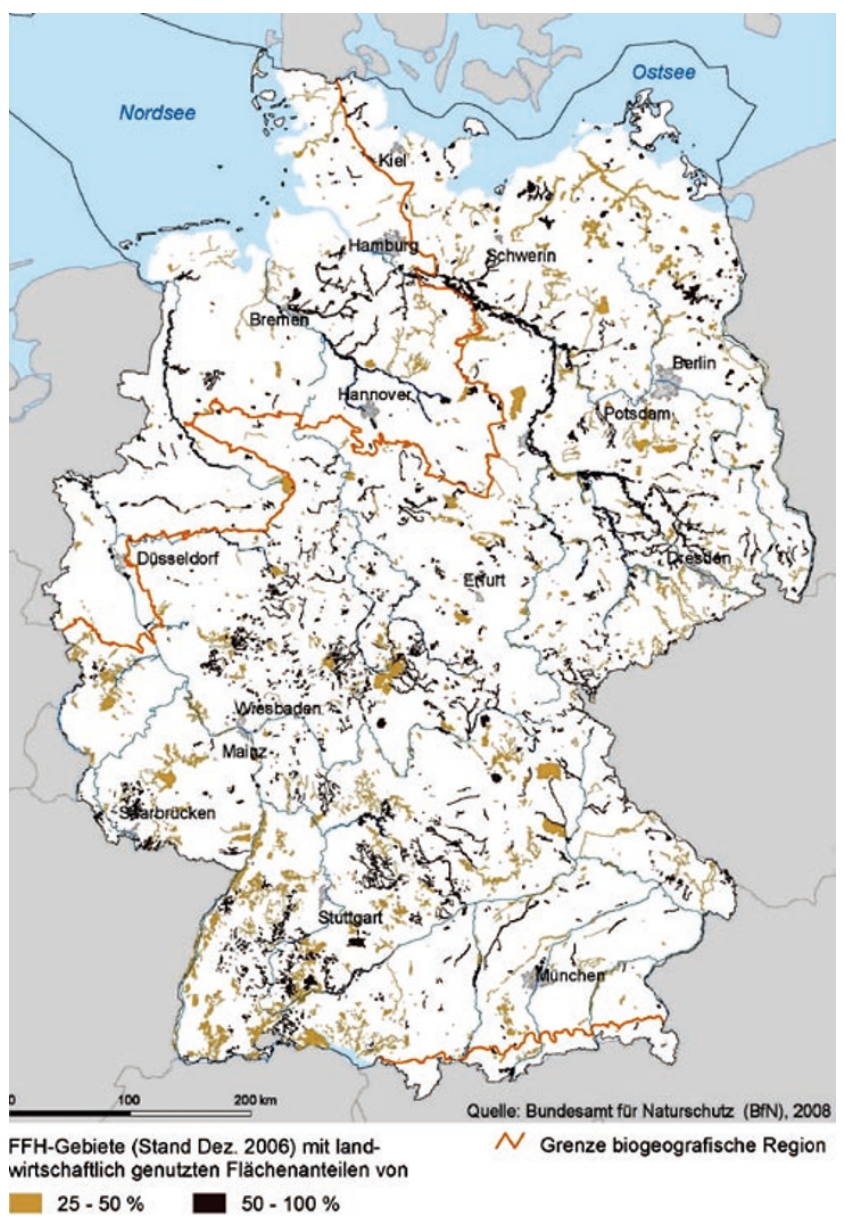

Abb. 2 FFH-Gebiete mit Anteilen der landwirtschaftlichen Nutzflächen von über $25 \%$ des Gebietes

(FFH-VP Art. 6, Abs. 3 und 4) beinhaltet. Der Erhaltungszustand ist definiert und umfasst die Parameter Verbreitungsgebiet, Fläche, Struktur und Funktionen (einschl. charakteristische Arten) und Zukunftsaussichten (s. Abschn. 5).

Bundesweit haben wir $53 \%$ landwirtschaftliche Nutzflächen mit einer Aufteilung in knapp zwei Drittel Ackerland und mehr als ein Drittel Grünland (Bundesamt für Naturschutz 2008). In den gemeldeten FFH-Gebieten liegt der Flächenanteil von landwirtschaftlichen Flächen deutlich niedriger bei ca. $13 \%$ Acker und 18\% Grünland (Raths et al. 2006) und einem relativ höheren Grünlandanteil.

Von den insgesamt gemeldeten 4.622 FFH-Gebieten enthalten 3.616 Gebiete die Habitatklassen (gemäß FFH-RL) „trockenes Grünland“, „mittleres und Feuchtgrünland“, „Intensivgrünland“ und „Acker") und ein knappes Viertel der Gebiete keine landwirtschaftlichen Flächen. In reichlich $24 \%$ der FFH-Gebiete sind über $50 \%$ landwirtschaftliche Nutzfläche vorhanden, weitere $18 \%$ der Gebiete enthalten zwischen 25 und $50 \%$ landwirtschaftliche Nutzflächen (Abb. 2). Klare Schwerpunkträume der FFH-Gebiete mit hohen Anteilen landwirtschaftlicher Nutzflächen liegen in Südwestdeutschland und in Teilen Nordostdeutschlands mit geringeren Anteilen in den meisten Mittelgebirgsregionen.

In den Ackerflächen liegen keine Lebensraumtypen des Anhangs I, obwohl die Roten Listen Deutschlands für die Biotope (Riecken et al. 2006) und die Pflanzengesellschaften (Rennwald 2000) hohe Gefährdungen für extensive Äcker und z. B. Pflanzengesellschaften des Caucalidion auf Kalkscherbenäckern in Wärmegebieten nachweisen und hier ein nationales Umsetzungsdefizit des Naturschutzes besteht.

Im Grünland lässt sich ebenfalls feststellen, dass nur verhältnismäßig wenige Lebensraumtypen oder solche mit geringen Anteilen an der Produktionsfläche genannt sind.

- Intensivgrünland (z. B. 3-4 Schnitte, Silagemahd, hohe Düngung usw.), sowie reguläre Weiden fallen nicht unter den Schutz der FFH-Richtlinie.

- Beim mittelintensiv genutzten Grünland, z. B. artenreiche Mähwiesen, fallen im wesentlichen die in Tabelle $1 \mathrm{zu}-$ sammengestellten LRT unter den Schutz des Anhangs I. Weiden, auch artenreiche und gefährdete Ausbildungen sind derzeit nicht vom Schutz des Anhangs I der FFHRichtlinie erfasst.

- Der Schwerpunkt des Schutzes nach Anhang I der FFHRichtlinie liegt bei den extensiv genutzten landwirtschaftlichen Flächen des Grünlandes und der Heiden, wie sie in Tabelle 2 dargestellt sind. Hier ist es oft so, dass die extensive landwirtschaftliche Nutzung naturschutzfachlich erwünscht, aber nicht mehr in allen Fällen rentabel umsetzbar ist, ohne für Management und Pflege finanzielle Unterstützung zu gewähren.

Die Lebensraumtypen mit mittelintensiv genutztem Grünland zeigen in Deutschland eine sehr klare regionale Verteilung und wurden in Abb. 3 mit ihren gemeldeten Flächengrößen klassifiziert. Dabei wurden jeweils die FFH-Gebiete dargestellt, die entsprechende Mindestgrößen dieser Lebensraumtypen (Summe der Typen der Kategorie I) enthalten. Nennenswerte Flächen mit über 100 ha je FFH-Gebiet dieser Lebensraumtypen finden sich nur in Südwestdeutschland und in den Mittelgebirgen, sie fehlen im nordwest- und nordostdeutschen Tiefland sowie im Alpenvorland fast ganz. Die Gesamtfläche dieser Lebensraumtypen des mittelintensiv genutzten Grünlandes beträgt mit knapp 97.000 ha bundesweit gerade mal $0,27 \%$.

Die Lebensraumtypen des extensiv genutzten Grünlandes und der Heiden umfassen nicht selten sehr kleinflächige, aber wegen ihrer hohen Gefährdung und historisch betrachtet oft sehr drastischen Flächenverluste für den Naturschutz dennoch sehr bedeutsame Restflächen. Daher wurden in Abb. 4 alle FFH-Gebiete dargestellt, die entsprechende Lebensraumtypen der Kategorie II enthalten. Hier sind jetzt die kleinen Restflächen von unter 10 ha dieser Lebensraumtypen in vielen FFH-Gebieten erkennbar. Um so schlimmer ist die Situation in Landschaftsräumen wie Teilen des Alpenvorlan- 


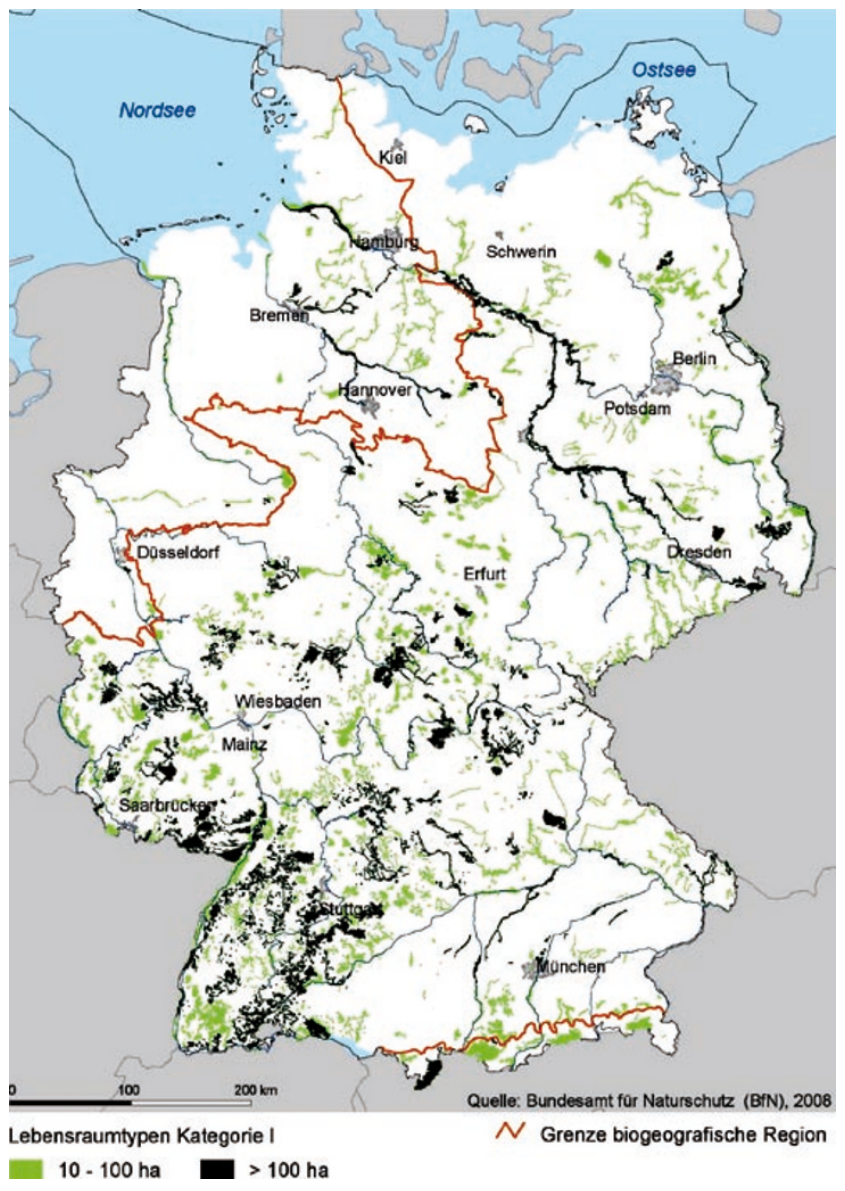

Abb. 3 FFH-Gebiete mit Anteilen mittelintensiven Grünlandes

des oder des nordwestdeutschen Tieflandes bzw. in weiten Teilen der atlantischen biogeografischen Region, wo noch nicht einmal schützenwerte Restflächen im Netz Natura 2000 vertreten sind (Entwicklungsbedarf v. a. auch im Sinne von Landschaftsleitbildern). Bei den Flächengrößen über 100 ha fallen jetzt auch die Salzrasen der Nord- und Ostseeküste und die wenigen noch großflächigen norddeutschen Heideflächen in ehemaligen oder noch genutzten militärischen Flächen auf. Die Gesamtfläche dieser Lebensraumtypen des extensiven Grünlandes und der Heiden beträgt knapp 280.000 ha und nimmt damit weniger als $0,8 \%$ der terrestrischen Bundesfläche ein. Zusammen nehmen alle landwirtschaftlich genutzten Flächen mit Lebensraumtypen des Anhangs I in Deutschland nur ca. $1 \%$ der Bundesfläche ein.

\section{Artenschutz}

In den Abb. 5-7 werden die wesentlichen Überlappungen der Vorkommen der Arten der Anhänge II, IV und V mit landwirtschaftlichen Nutzungstypen (Quelle: CORINE

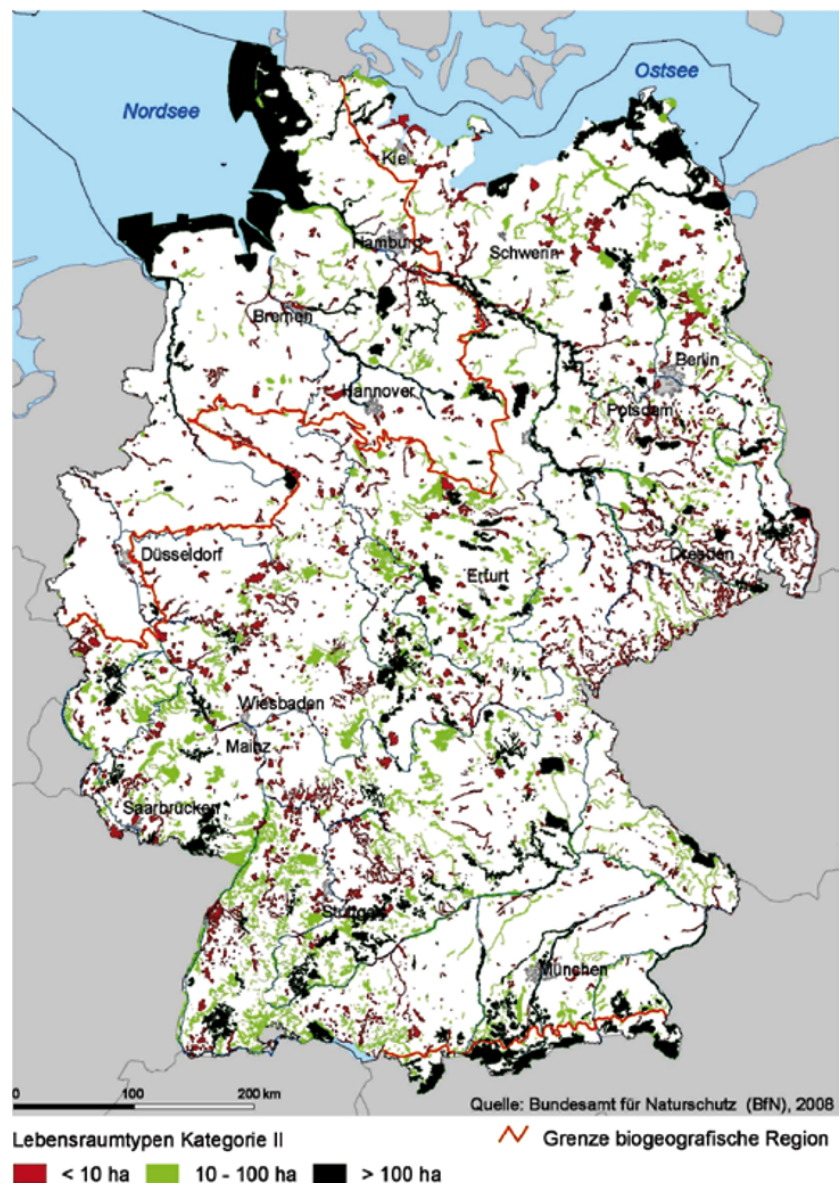

Abb. 4 FFH-Gebiete mit Anteilen extensiv genutzten Grünlandes und Heiden

Land Cover; Umweltbundesamt, DLR-DFD 2004) bzw. mit den Habitatklassen der FFH-Gebiete dargestellt. Abbildung 5 zeigt die aktuellen Weinanbaugebiete in Deutschland. Diese überlappen sich in vielen Regionen mit den Vorkommen der Mauereidechse (Podarcis muralis) und der Westlichen Smaragdeidechse (Lacerta bilineata). Verbreitungspunkte außerhalb der Weinbaugebiete beziehen sich überwiegend auf Felsen und Felsrasen, die keiner landwirtschaftlichen Nutzung unterliegen. In Abb. 6 werden entsprechend die Überlappungen von Grünland mit den an die Wirtspflanze Großer Wiesenknopf (Sanguisorba major) gebundenen Schmetterlingsarten Heller und Dunkler Wiesenknopf-Ameisenbläuling (Maculinea nausithous, Maculinea teleius) (Petersen et al. 2003) dargestellt. Hinzu kommt die Verbreitung der Äskulappnatter (Elaphe longissima), die in ihrem westlichen Verbreitungsgebiet in Deutschland (Odenwald und Rheingau-Taunus) bevorzugt Streuobstwiesen, Wiesenhänge und Weiden besiedelt (Petersen et al. 2004).

Abbildung 7 zeigt die Verbreitung der Dicken Trespe (Bromus grossus) als Art der Getreidefelder, vom Kugelhornmoos (Notothylas orbicularis) als einer Art besonders 


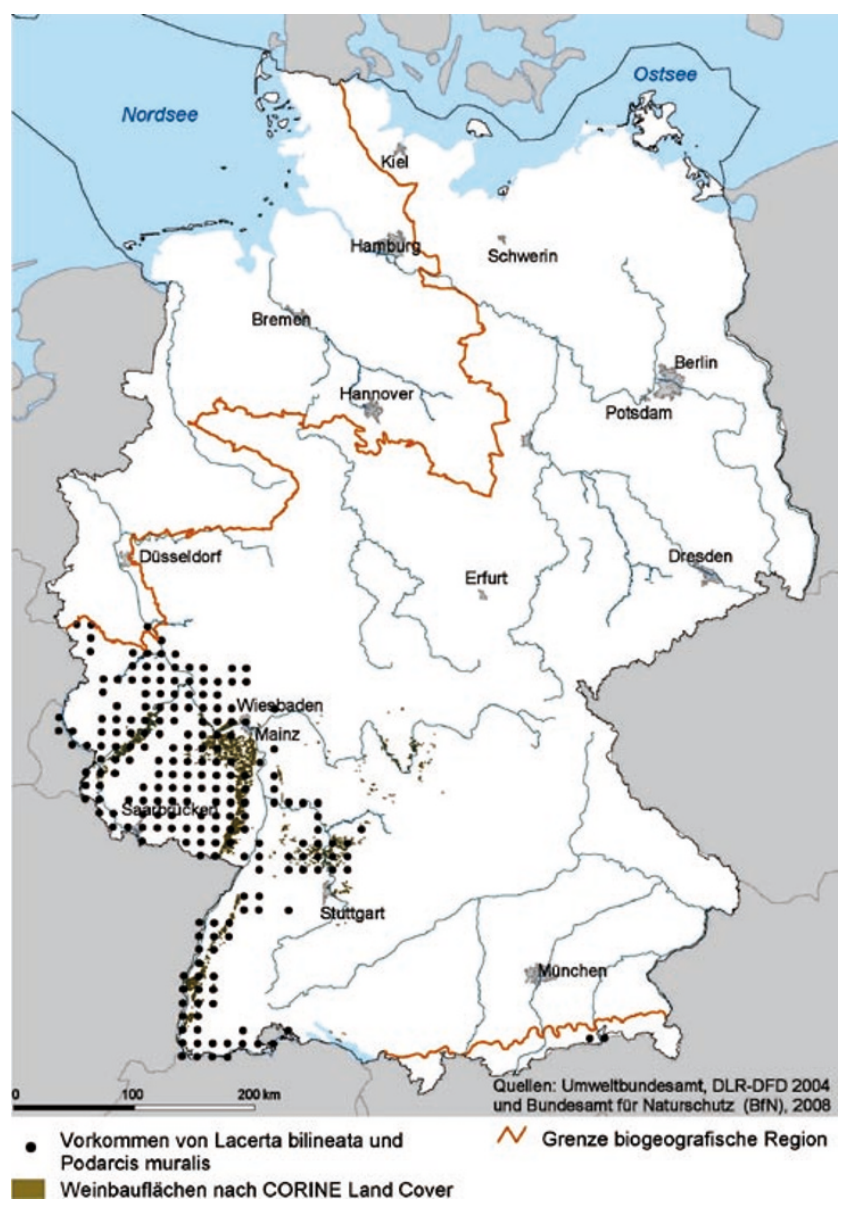

Abb. 5 Überlagerung von Weinbauflächen mit den Vorkommen typischer FFH-Arten der Weinberge (Vorkommen der Äskulapnatter in Bayern, die sich außerhalb landwirtschaftlicher Flächen befinden, sind in der Karte nicht dargestellt)

der Getreide- und Kartoffeläcker sowie des Feldhamsters (Cricetus cricetus). Die Verbreitungspunkte dieser Arten sind mit der Verbreitung der Knoblauchkröte (Pelobates fuscus) hinterlegt. Die Knoblauchkröte ist nicht so spezifisch an Äcker gebunden, wie die drei anderen Arten. Nach Untersuchungen von Schiemenz und Günther (1994) in Ostdeutschland wurde die Art in ihren Landlebensräumen zu $10,8 \%$ in Äckern und zu 10,7\% in Wiesen und Weiden gefunden. Die restlichen Funde beziehen sich auf Gärten und andere Siedlungsbereiche.

Alle aufgeführten Arten - mit Ausnahme des Mooses sind Arten des Anhanges IV der FFH-RL und unterliegen damit einem strengen Artenschutz. Da sie sich oft außerhalb von Schutzgebieten aufhalten, können nur spezielle Artenschutzprogramme und Managementkonzepte einen günstigen Erhaltungszustand der Arten gewährleisten. Hier sind die Länder mit Förderprogrammen in Kooperation mit den Nutzern gefordert, entsprechende Konzepte umzusetzen. Das gilt ebenfalls für den größten Teil der Arten der Kate-

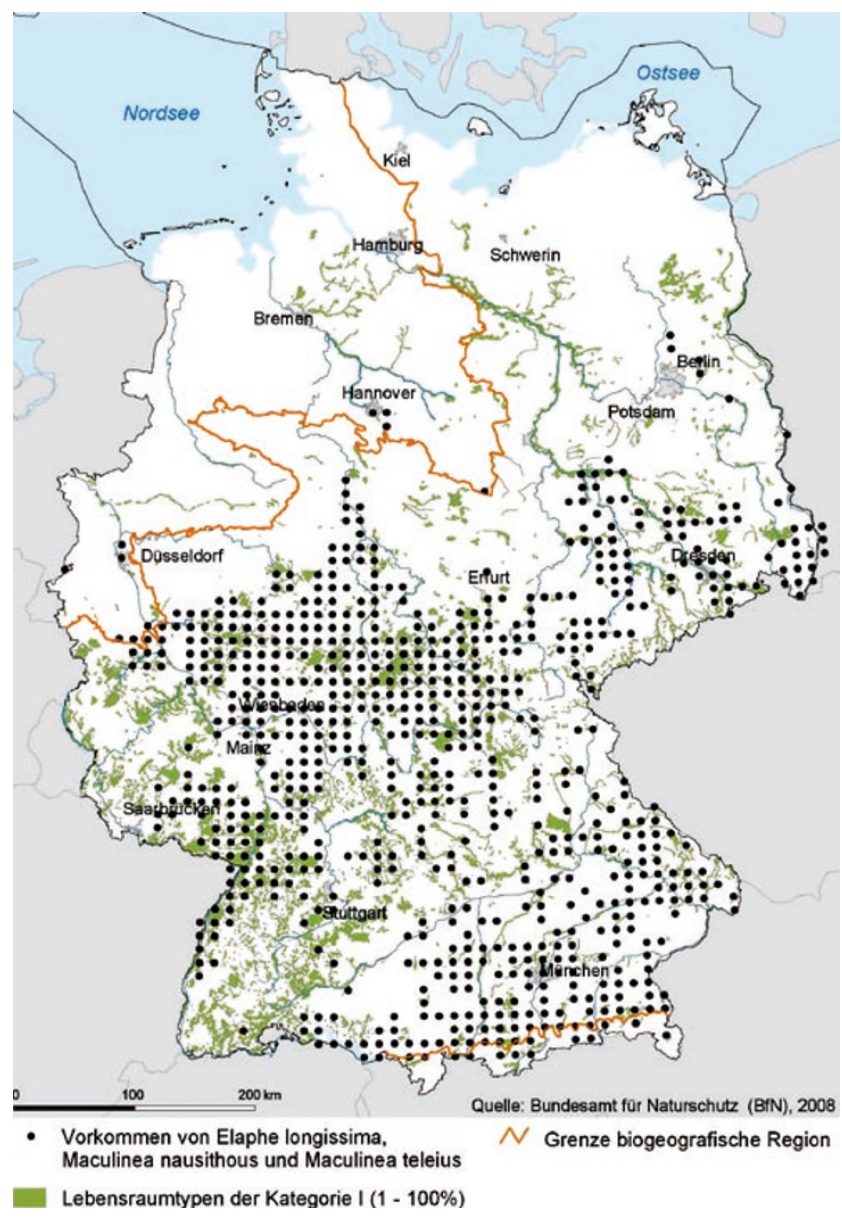

Abb. 6 FFH-Gebiete mit Anteilen mittelintensiven Grünlandes und den Vorkommen typischer Grünlandarten der FFH-RL

gorie II, die die extensiv genutzte Agrarlandschaft besiedeln und z. T. auch für Arten der Kategorie III, die indirekt durch den Agrarbereich betroffen sind.

\section{Bewertung des Erhaltungszustandes in der Agrar- landschaft - Ergebnisse des nationalen Berichts}

\subsection{Hintergrund}

Ein zentrales Ziel der FFH-Richtlinie ist die dauerhafte Sicherung eines ,günstigen Erhaltungszustandes“ für die auf dem Anhang I genannten Lebensraumtypen und die auf den Anhängen II, IV und V genannten Arten. Durch ein europaweites kohärentes Schutzgebietssystem und durch entsprechende, durch die Mitgliedstaaten zu ergreifende Schutz- und Entwicklungsmaßnahmen, soll dieses Ziel erreicht werden.

Die FFH-Richtlinie schreibt im Artikel 17 eine regelmäBige und umfassende Berichterstattung über den Erhaltungszustand aller Lebensraumtypen und Arten der Richtlinie vor. Alle Mitgliedstaaten müssen im sechsjährigen Turnus 


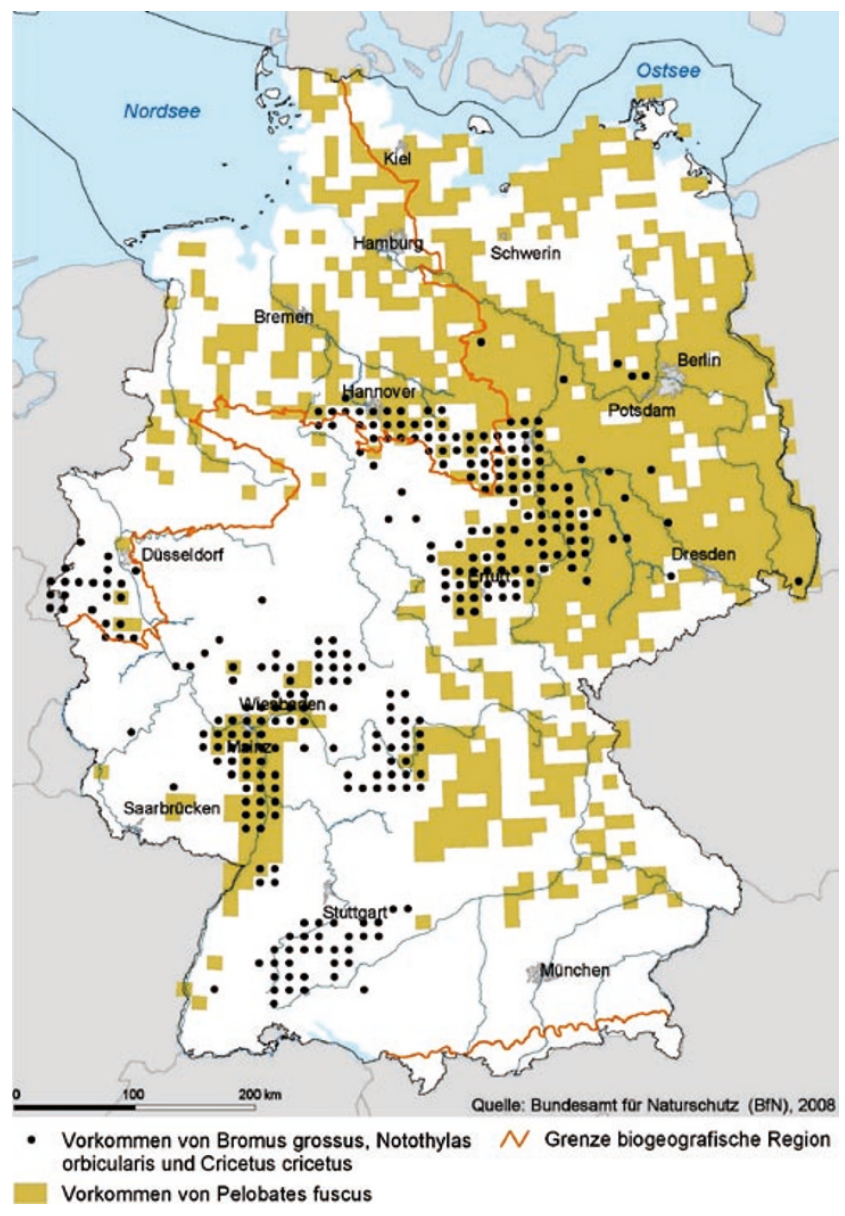

Abb. 7 Verbreitung typischer Ackerarten der FFH-RL

über diesen Erhaltungszustand in den einzelnen biogeografischen Regionen berichten. Die letzte Berichtsperiode betrachtet den Zeitraum 2001 bis 2006.

Der Erhaltungszustand wird bei den Arten über die vier Parameter „Aktuelles Verbreitungsgebiet“, „Population“, „Habitat der Art“ und „Zukunftsaussichten“ bewertet. Bei den Lebensraumtypen erfolgt die Bewertung über die Parameter „Aktuelles Verbreitungsgebiet“, „Aktuelle Fläche“, „Spezifische Strukturen und Funktionen“ und „Zukunftsaussichten". Jeder einzelne Parameter wird pro biogeografische Region art- und lebensraumtypenspezifisch mit einem dreistufigen System (günstig, ungünstig - unzureichend, ungünstig - schlecht) bewertet. Bei defizitärer Datenlage kann ein Parameter auch als unbekannt bewertet werden. Anhand festgelegter Kriterien wird aus den Bewertungen der vier Einzelparameter ein Gesamtwert für jede biogeografische Region ermittelt (Balzer et al. 2008). Eine Übersicht über die Bewertung des Erhaltungszustandes der Lebensraumtypen und Arten (summarisch) findet sich in Ellwanger et al. (2008) und auch auf der Homepage des BfN (Nationaler Bericht 2007).
Durch die EU-Kommission wurden die Berichte der Mitgliedstaaten zusammengefasst, sodass schließlich für jede Art und jeden Lebensraumtyp eine Bewertung pro betroffene biogeografische Region angegeben werden kann. Deutschland hat Anteil an drei der insgesamt neun biogeografischen Regionen der EU 27 (s. Abb. 1).

\subsection{Bewertung der Arten und LRT}

Insgesamt wurden in Deutschland für den Nationalen Bericht 230 Arten der Anhänge II, IV und V bewertet. Die Hauptlebensräume bzw. überlebenswichtige Teillebensräume von elf der bewerteten Arten liegen überwiegend in Flächen, die landwirtschaftlich meist „mittelintensiv“ genutzt werden. In der alpinen und atlantischen Region wurden $55 \%$ dieser Arten EU-weit mit einem ungünstigen bzw. schlechten Erhaltungszustand bewertet, in der kontinentalen Region sind es sogar $73 \%$ (Tabelle 4). In Deutschland wurde der Erhaltungszustand aller durch landwirtschaftliche Nutzung stark betroffener Arten mit Ausnahme von Myotis nattereri und Maculinea nausithous (alpine Region) ungünstig bis schlecht eingeschätzt. Für Bromus grossus, die in Deutschland nur in der kontinentalen Region vorkommt, konnte aufgrund der defizitären Datenlage keine Bewertung durchgeführt werden.

Am Beispiel des Dunklen Wiesenknopf-Ameisenbläulings (Maculinea nausithous) ist erkennbar, dass der Erhaltungszustand einer im extensiven Gründland lebenden Art direkt von der Bewirtschaftung der Flächen abhängig ist. In der alpinen Region ist die landwirtschaftliche Nutzung in vielen Bereichen schon aufgrund der Topografie extensiver als beispielsweise in der atlantischen und kontinentalen Region. Der Erhaltungszustand der Art in der alpinen Region wurde daher deutlich günstiger eingeschätzt als z. B. in der atlantischen Region. Dieser Trend spiegelt sich auch in der EU-weiten Bewertung wieder: hier wurde der Bläuling in der alpinen Region „gelb“ bewertet, in der atlantischen und kontinentalen Region jedoch „rot“ (http://biodiversity.eionet.europa.eu/article17).

Insgesamt wurden in Deutschland 91 Lebensraumtypen des Anhangs I bewertet. Unter den Lebensraumtypen finden sich drei Typen die landwirtschaftlich genutzt werden. Die Bewertung des Erhaltungszustandes dieser LRT ist deutschlandweit und EU-weit über alle drei Regionen hinweg durchgängig ungünstig bis schlecht (Tabelle 5).

Die negative Bewertung der Arten und Lebensraumtypen, die durch landwirtschaftliche Nutzung betroffen sind, ist größtenteils darauf zurückzuführen, dass die Nutzung der Flächen nicht dem Lebenszyklus der Arten bzw. dem Pflegebedarf der Lebensraumtypen angepasst ist. Viele dieser Arten und Lebensraumtypen sind auf eine landwirtschaftliche Nutzung angewiesen. Nutzungsaufgabe führt vielerorts zur Verbrachung der Flächen und letztendlich zum Verschwin- 
Tabelle 4 Bewertung der FFH-Arten, die durch mittelintensive landwirtschaftliche Nutzung betroffen sind, gemäß des Nationalen Berichtes (Artikel 17 FFH-RL)

\begin{tabular}{|c|c|c|c|c|c|c|c|c|c|c|}
\hline \multirow[t]{2}{*}{$\begin{array}{l}\text { Wiss. } \\
\text { Artname }\end{array}$} & \multirow[t]{2}{*}{$\begin{array}{l}\text { Artname } \\
\text { deutsch }\end{array}$} & \multicolumn{3}{|c|}{ Alpine Region } & \multicolumn{3}{|c|}{ Atlantische Region } & \multicolumn{3}{|c|}{ Kontinentale Region } \\
\hline & & $\begin{array}{c}\text { Bewertung } \\
\text { D }\end{array}$ & $\begin{array}{c}\text { Anz. } \\
\text { Staaten }\end{array}$ & $\begin{array}{c}\text { Bewertung } \\
\text { EU }\end{array}$ & $\begin{array}{c}\text { Bewertung } \\
\text { D }\end{array}$ & $\begin{array}{c}\text { Anz. } \\
\text { Staaten }\end{array}$ & $\begin{array}{c}\text { Bewertung } \\
\text { EU }\end{array}$ & $\begin{array}{c}\text { Bewertung } \\
\text { D }\end{array}$ & $\begin{array}{c}\text { Anz. } \\
\text { Staaten }\end{array}$ & $\begin{array}{l}\text { Bewer- } \\
\text { tung EU }\end{array}$ \\
\hline $\begin{array}{l}\text { Bromus } \\
\text { grossus }\end{array}$ & Dicke Trespe & - & 1 & $\mathrm{XX}$ & - & 0 & - & $\mathrm{XX}$ & 3 & $\mathrm{XX}$ \\
\hline $\begin{array}{l}\text { Cricetus } \\
\text { cricetus }\end{array}$ & Feldhamster & - & 1 & U1 & $\mathrm{U} 2$ & 3 & $\mathrm{U} 2$ & $\mathrm{U} 2$ & 7 & $\mathrm{U} 2$ \\
\hline $\begin{array}{l}\text { Elaphe longis- } \\
\text { sima }\end{array}$ & Äskulapnatter & U1 & 8 & $\mathrm{XX}$ & - & 2 & U1 & $\mathrm{U} 2$ & 6 & U1 \\
\hline $\begin{array}{l}\text { Lacerta bili- } \\
\text { neata }\end{array}$ & $\begin{array}{c}\text { Westl. Smaragd- } \\
\text { eidechse }\end{array}$ & - & 6 & U1 & - & 1 & $\mathrm{XX}$ & $\mathrm{U} 2$ & 6 & $\mathrm{U} 2$ \\
\hline $\begin{array}{l}\text { Maculinea } \\
\text { nausithous }\end{array}$ & $\begin{array}{l}\text { Dunkler Wiesen- } \\
\text { knopf-Ameisen- } \\
\text { bläuling }\end{array}$ & FV & 5 & $\mathrm{U} 1$ & $\mathrm{U} 2$ & 3 & $\mathrm{U} 2$ & $\mathrm{U} 1$ & 6 & $\mathrm{U} 2$ \\
\hline $\begin{array}{l}\text { Maculinea } \\
\text { teleius }\end{array}$ & $\begin{array}{l}\text { Heller Wiesen- } \\
\text { knopf-Ameisen- } \\
\text { bläuling }\end{array}$ & U1 & 7 & $\mathrm{U} 2$ & - & 2 & $\mathrm{U} 2$ & U1 & 7 & $\mathrm{U} 2$ \\
\hline $\begin{array}{l}\text { Myotis nat- } \\
\text { tereri }\end{array}$ & $\begin{array}{c}\text { Fransenfleder- } \\
\text { maus }\end{array}$ & $\mathrm{FV}$ & 8 & $\mathrm{XX}$ & $\mathrm{FV}$ & 9 & $\mathrm{XX}$ & FV & 11 & $\mathrm{U} 1$ \\
\hline $\begin{array}{l}\text { Notothylas } \\
\text { orbicularis }\end{array}$ & Kugel-Hornmoos & - & 1 & $\mathrm{U} 2$ & - & 0 & - & $\mathrm{U} 2$ & 2 & $\mathrm{U} 2$ \\
\hline $\begin{array}{l}\text { Pelobates } \\
\text { fuscus }\end{array}$ & Knoblauchkröte & - & 2 & U1 & U1 & 5 & U1 & U1 & 8 & $\mathrm{U} 1$ \\
\hline $\begin{array}{l}\text { Plecotus aust- } \\
\text { riacus }\end{array}$ & Graues Langohr & - & 7 & $\mathrm{XX}$ & U1 & 7 & $\mathrm{XX}$ & U1 & 9 & $\mathrm{XX}$ \\
\hline $\begin{array}{l}\text { Podarcis } \\
\text { muralis }\end{array}$ & Mauereidechse & U1 & 7 & $\mathrm{FV}$ & U1 & 4 & $\mathrm{FV}$ & U1 & 8 & $\mathrm{FV}$ \\
\hline
\end{tabular}

Legende zur Bewertung des Erhaltungszustandes: FV günstig, U1 ungünstig unzureichend, U2 ungünstig schlecht, XX unbekannt

Tabelle 5 Bewertung der FFH-LRT, die durch mittelintensive landwirtschaftliche Nutzung betroffen sind, gemäß des Nationalen Berichtes (Artikel 17 FFH-RL). Legende siehe Tabelle 4

\begin{tabular}{|c|c|c|c|c|c|c|c|c|c|}
\hline \multirow[t]{2}{*}{ Lebensraumtyp } & \multicolumn{3}{|c|}{ Alpine Region } & \multicolumn{3}{|c|}{ Atlantische Region } & \multicolumn{3}{|c|}{ Kontinentale Region } \\
\hline & $\begin{array}{c}\text { Bewertung } \\
\text { D }\end{array}$ & $\begin{array}{l}\text { Anz. } \\
\text { Staaten }\end{array}$ & $\begin{array}{l}\text { Bewer- } \\
\text { tung EU }\end{array}$ & $\begin{array}{l}\text { Bewer- } \\
\text { tung D }\end{array}$ & $\begin{array}{l}\text { Anz. } \\
\text { Staaten }\end{array}$ & $\begin{array}{l}\text { Bewer- } \\
\text { tung EU }\end{array}$ & $\begin{array}{l}\text { Bewer- } \\
\text { tung D }\end{array}$ & $\begin{array}{l}\text { Anz. } \\
\text { Staaten }\end{array}$ & $\begin{array}{c}\text { Bewer- } \\
\text { tung EU }\end{array}$ \\
\hline $\begin{array}{l}\text { 6440: Brenndolden-Auen- } \\
\text { wiesen }\end{array}$ & - & 0 & - & $\mathrm{U} 2$ & 1 & $\mathrm{U} 2$ & $\mathrm{U} 2$ & 5 & $\mathrm{U} 2$ \\
\hline $\begin{array}{l}\text { 6510: Magere Flachland- } \\
\text { Mähwiesen }\end{array}$ & $\mathrm{U} 2$ & 8 & U1 & $\mathrm{U} 2$ & 8 & U2 & U1 & 10 & U1 \\
\hline 6520: Berg-Mähwiesen & U1 & 9 & $\mathrm{U} 2$ & - & 1 & U2 & U1 & 8 & U1 \\
\hline
\end{tabular}

den des Lebensraumtyps bzw. der Arten. Die drei genannten Lebensraumtypen können durch eine extensive Mahd bzw. bei den Brenndolden-Auenwiesen auch Beweidung genutzt und gleichzeitig gepflegt werden. Gleiches trifft auch für die beiden Bläulingsarten Maculinea nausithous und M. teleius zu, deren Habitate sich oft im bewirtschafteten Grünland befinden.

Unter den Arten, die im Rahmen des Nationalen Berichtes bewertet wurden, befinden sich weiterhin 36 Arten, de- ren Hauptlebensraum bzw. überlebenswichtige Teillebensräume in meist landwirtschaftlich extensiv bewirtschafteten Bereichen liegen. Die deutschlandweite Bewertung dieser Arten wird in Abb. 8 dargestellt.

Unter den im Rahmen des Nationalen Berichtes bewerteten Lebensraumtypen befinden sich 17 Typen, die landwirtschaftlich extensiv genutzt werden. Abbildung 9 gibt Auskunft über die deutschlandweite Bewertung dieser Lebensraumtypen. 


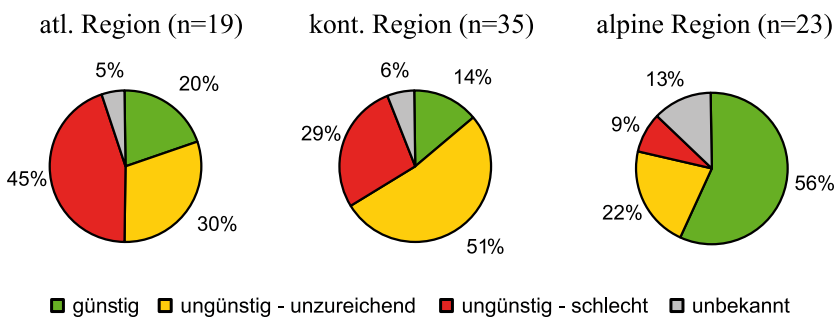

Abb. 8 Bewertung der FFH-Arten, die durch extensive landwirtschaftliche Nutzung betroffen sind, gemäß des Nationalen Berichtes (Artikel 17 FFH-RL)

Im Vergleich zu den LRT und Arten, die durch mittelintensive landwirtschaftliche Bewirtschaftung betroffen sind, fällt die deutschlandweite Bewertung der Arten, die durch extensive landwirtschaftliche Bewirtschaftung betroffen sind, deutlich besser aus. Es gibt in jeder biogeografischen Region mehrere Arten und mindestens einen LRT, deren Erhaltungszustand als günstig bewertet wurde. Eine gut abgestimmte extensive landwirtschaftliche Nutzung kann, wie bereits oben erwähnt, für den Erhalt und die Pflege von Arten und Lebensraumtypen sehr wichtig und hilfreich sein.

Bei der Betrachtung des Erhaltungszustandes im Zusammenhang mit der landwirtschaftlichen Nutzung muss jedoch auch berücksichtigt werden, dass es Arten gibt, die trotz bester Bewirtschaftung der Flächen einen schlechten Erhaltungszustand haben. Hier liegen die Defizite oft darin, dass die besiedelten Flächen zu weit auseinander liegen, sodass es keinen Austausch zwischen den (Teil-)Populationen mehr gibt. In diesen Fällen müssen zum Erhalt der Art weitergehende Maßnahmen ergriffen werden (Entfernung unüberwindbarer Barrieren, Schaffung von Ausbreitungskorridoren o. ä.), die den Populationsaustausch wieder ermöglichen.

\section{Ausblick}

Wenngleich die Landwirtschaft flächenmäßig von den Lebensraumtypen und Arten der Anhänge der FFH-Richtlinie nur wenig betroffen ist und intensiv genutzte Flächen nicht direkt betroffen sind, gibt es eine Reihe indirekter Wirkungen, die erheblichen Einfluss auf die Entwicklung der Erhaltungszustände haben können.

In der alpinen und atlantischen Region wurden $55 \%$ der Arten mit Hauptvorkommen in mittelintensiv genutzten Agrarflächen EU-weit mit einem ungünstigen bzw. schlechten Erhaltungszustand bewertet, in der kontinentalen Region sind es sogar $73 \%$. Die drei Lebensraumtypen, die von einer mittelintensiven Landwirtschaft betroffen sind, wurden im Erhaltungszustand alle als ungünstig (unzureichend bis schlecht) bewertet. Hier besteht dringender Handlungsbe- atl. Region $(\mathrm{n}=15)$

kont. Region $(\mathrm{n}=16)$

alpine Region $(\mathrm{n}=7)$
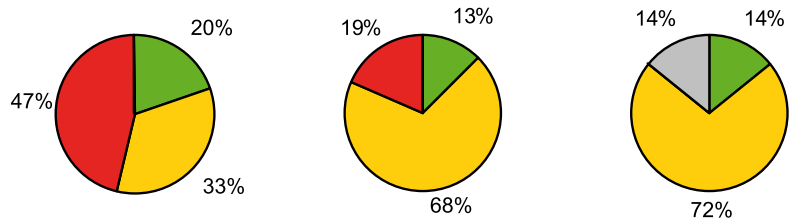

$\square$ günstig $\square$ ungünstig - unzureichend

$\square$ ungünstig - schlecht

Abb. 9 Bewertung der FFH-LRT, die durch extensive landwirtschaftliche Nutzung betroffen sind, gemäß des Nationalen Berichtes (Artikel 17 FFH-RL)

darf, vor dem Hintergrund, dass eine Bewirtschaftung bzw. Pflege für den Erhalt erforderlich ist, aber gleichzeitig wegen nicht adäquater Formen oder Intensität der Nutzung das Risiko einer weiteren Verschlechterung besteht.

Wesentliche Gefährdungsfaktoren und Ursachen für einen ungünstigen Erhaltungszustand vieler Lebensraumtypen sind Nährstoffeinträge, die sowohl durch direktes Ausbringen von Dünger, indirekten Eintrag aus benachbarten Flächen als auch durch Luftstickstoffeinträge auftreten (Bobbink 1998). Hier besteht, wie ja auch aus der Trinkwassergewinnung und Wasserreinhaltung bekannt, ein erhebliches Konfliktpotenzial mit intensiv genutzten landwirtschaftlichen Flächen im unmittelbaren Umfeld von FFH-Gebieten mit Lebensraumtypen, die oligotrophe Verhältnisse benötigen. So lag z. B. allein der durchschnittliche atmosphärische Gesamtstickstoffeintrag im Jahr 1999 bei etwa $26,4 \mathrm{~kg} / \mathrm{ha}$, mit den höchsten Depositionsraten von über $80 \mathrm{~kg} / \mathrm{ha}$ Jahr (Gauger et al. 2002).

Demgegenüber stehen kritische Grenzwerte (critical loads) der Belastbarkeit von Ökosystemen und Biotoptypen wie sie z.B. im ICP-Mapping-Manual der UNECE Convention on long-range transboundary Air Pollution (ICP Modelling and Mapping 2004) angegeben werden. Dort wurden tabellarisch die Ökosystemtypen bzw. die Lebensraumklassifizierung des Europäischen EUNIS-Systems mit Ihren Grenzwerten angegeben, die sich in den allermeisten Fällen direkt den FFH-Lebensraumtypen des Anhangs I zuordnen lassen. Danach liegen die critical loads z.B. bei Mooren und feuchten Dünentälern in der Größenordnung von $5-10 \mathrm{~kg} \mathrm{~N} / \mathrm{ha} / \mathrm{J}$, bei vielen LRT der extensiven Kulturlandschaften (z.B. Heiden, viele Grünlandtypen) sowie bei den mitteleuropäischen Wäldern im Regelfall zwischen $10-20 \mathrm{~kg} \mathrm{~N} / \mathrm{ha} / \mathrm{J}$. Aus einem Vergleich der critical loads mit den Gesamtstickstoffdepositionen lassen sich deutschlandweit Karten der Überschreitung der kritischen Grenzwerte berechnen (Öko-Data 2000). Danach sind die Grenzwerte bei über $90 \%$ der Flächen überschritten, in großen Regionen Deutschlands, v. a. im westdeutschen Tiefland, in weiten Teilen des Alpenvorlandes und in den östlichen Mittelgebirgen z. B. in Sachsen und Bayern sogar um über $25 \mathrm{~kg} / \mathrm{ha} / \mathrm{J}$. Ein 
erheblicher Teil dieser Eutrophierungsbelastung stammt aus landwirtschaftlichen Flächen, infolge hoher Düngung, Intensivweide, Rinderhaltung oder Gülleausbringung. Hauptverursacher zu rund $95 \%$ der Stickstoffemissionen durch Ammoniak ist die Landwirtschaft (Bayerisches LfU: http:// www.lfu.bayern.de/umweltwissen/doc/uw_6_ammoniak_ ammonium.pdf). Dies liegt in der gleichen Größenordnung wie die Gesamtsumme der emittierten Stickstoffoxide (CO, $\mathrm{CO}_{2}$ ). Dabei entfallen nach Döhler et al. (2002) rund 50\% davon auf Rinderhaltung (Ställe, Gülle, Jauche usw.), weitere rund $20 \%$ jeweils auf Schweinehaltung und auf Emissionen durch Mineraldünger.

Hier sind dringend Maßnahmen erforderlich, da sich sonst der Erhaltungszustand zahlreicher Lebensraumtypen in diesen Regionen künftig weiter verschlechtern wird oder bei schlechtem Erhaltungszustand keine Verbesserungen zu erwarten sind. Mittelbar sind dort weite Teile des Natura 2000 Netzes betroffen, auch wenn auf diesen Flächen selbst keine landwirtschaftliche Nutzung stattfindet, und kann die Einhaltung des Verschlechterungsverbotes nach Art. 6 Abs. 2 der FFH-Richtlinie ein erhebliches Problem darstellen. In Dänemark sind die critical loads bei der Erhaltungszustandsbewertung nach FFH-Richtlinie für die Bewertung der Zukunftsaussichten und in Maßnahmenplanungen direkt eingegangen. Dies wäre auch eine Option für die deutsche und die europäische Bewertung.

Ein weiteres unübersehbares Konfliktfeld zwischen dem EU-Naturschutz und der Landwirtschaft besteht im flächendeckenden oder großräumigen Einsatz von Bioziden mit direkten oder indirekten negativen Auswirkungen auf die europaweit geschützten Arten und Lebensraumtypen in den Natura 2000 Gebieten. Ein Beispiel ist die Bekämpfung des als invasivem Schädling eingestuften Westlichen Maiswurzelbohrers (Diabrotica virgifera virgifera) in der Oberrheinebene in Baden-Württemberg. 1992 erstmals in Europa in Jugoslawien eingeschleppt, trat 2007 Schadbefall in Baden-Württemberg und in Bayern auf. Die Anwendung von mit Clothianidin gebeiztem Mais hat im Rheintal im Mai/Juni 2008 zum Absterben von rund 20.000 Bienenvölkern geführt, die laut Untersuchungen des Justus Kühn-Insituts eindeutig auf diesen Gifteinsatz zurückgehen und auch außerhalb der unmittelbaren Ausbringungsbereiche lagen (z. B. Pressemitteilung vom 16.5.2008, http://idw-online.de/ pages/de/news260637). Danach wurde die Anwendung vorübergehend verboten und inzwischen teilweise wieder zugelassen. Angesicht der hohen Neurotoxizität schon in geringen Konzentrationen und der Breitbandwirkung auf andere Insektengruppen ist davon auszugehen, dass hier in angrenzenden Natura 2000 Gebieten auch einheimische Wildbienen, Schmetterlinge usw. betroffen sind. Sowohl die (Wieder-) Zulassung solcher Mittel für flächenmäßigen Einsatz mit möglichem negativen Auswirkungen auf Natura 2000 Gebiete (charakteristische Arten der Lebensraumtypen, sowie Arten der Anhänge II und IV), als auch die konkrete Anwendung im möglichen Einflussbereich von Natura 2000 Gebieten Bedarf einer FFH-Verträglichkeitsprüfung (Plan- bzw. Projekt-VP, die offensichtlich nicht durchgeführt wurde). Bei einer erforderlichen Alternativenprüfung wäre zudem deutlich geworden, dass den Erfordernissen der EU-Richtlinie 2000/29/EG (Der Rat der Europäischen Union 2000) zur Bekämpfung von Schadorganismen durch eine entsprechende Einhaltung mehrjähriger Fruchtfolgen weitaus besser entsprochen werden könnte, wie dies mit Erfolg z.B. in der Schweiz durchgeführt wird. Darüber hinaus ist davon auszugehen, dass auch streng geschützte Anhang IVArten geschädigt wurden und damit auch artenschutzrechtliche Ausnahmegenehmigungen notwendig gewesen wären.

Zusätzliche Bereiche der Landwirtschaft, die negative Auswirkungen auf das Netz Natura 2000 und die Erhaltungszustände der europaweit geschützten Arten und Lebensraumtypen haben können, sind der zunehmende Anbau von nachwachsenden Rohstoffen (so stieg von 2004 bis 2006 die Anbaufläche bereits von 13.500 auf rund 287.000 ha (Bundesamt für Naturschutz 2008). Weitere Konfliktfelder sind der Einsatz von Gentechnisch Veränderten Organismen (GVO) mit seinen direkten und indirekten Auswirkungen und ein zunehmender Nutzungswandel, der sich in einer immer größer werden Schere zwischen Intensivierung hochproduktiver Flächen und einer Aufgabe extensiver Flächen ausdrückt. Die Funktionalität des Netzes Natura 2000 ist in hohem Maße auch von Wechselbeziehungen abhängig, wie Wanderung, Genaustausch usw., die ein Mindestmaß an Biotopverbundflächen oder Eignung der umgebenden Landschaft für die bedrohten Tier- und Pflanzenarten voraussetzen. Neben bundesweiten und landesweiten Konzeptionen zum Biotopverbund und Workshops zur Umsetzung der Kohärenz nach Art. 10 FFH-Richtlinie (http://www.bfn.de/0316_kohaerenz.html), laufen hier auch Entwicklungs- und Erprobungsvorhaben des Bundesamts für Naturschutz, um modellhaft die Umsetzung in der Agrarlandschaft zu fördern (z. B. F+E-Projekt zur Biologischen Vielfalt in der Agrarlandschaft im Raum Zwillbrock).

\section{Literatur}

Balzer S, Ellwanger G, Raths U, Schröder E, Ssymank A (2008) Verfahren und erste Ergebnisse des nationalen Berichts nach Artikel 17 der FFH-Richtlinie. Natur Landsch 83(3):111-117

Bayerisches LfU: http://www.lfu.bayern.de/umweltwissen/doc/uw_6_ ammoniak_ammonium.pdf (4.8.2008)

Bobbink R, Hornung M, Roelofs JGM (1998) The effects of air-borne nitrogen pollutants on species diversity in natural and semi-natural European vegetation. J Ecol 86:717-738

Bundesamt für Naturschutz (2008) Daten zur Natur 2008. Landwirtschaftsverlag, Münster

CORINE Land Cover (2004) Umweltbundesamt, DLR-DFD

Der Rat der Europäischen Gemeinschaften (1992) Richtlinie 92/43/ EWG des Rates vom 21. Mai 1992 zur Erhaltung der natürlichen 
Lebensräume sowie der wildlebenden Tiere und Pflanzen. Amtsblatt der Europäischen Gemeinschaften, Reihe L 206:7-50

Der Rat der Europäischen Union (2000) Richtlinie 2000/29/EG des Rates vom 8. Mai 2000 über Maßnahmen zum Schutz der Gemeinschaft gegen die Einschleppung und Ausbreitung von Schadorganismen der Pflanzen und Pflanzenerzeugnisse. Amtsblatt der Europäischen Gemeinschaften Nr. L 169 vom 10. Juli

Der Rat der Europäischen Union (2006) Richtlinie 2006/105/EG des Rates vom 20. November 2006 zur Anpassung der Richtlinien 73/239/EWG, 74/557/EWG und 2002/83/EG im Bereich Umwelt anlässlich des Beitritts Bulgariens und Rumäniens. Amtsblatt der Europäischen Union, Reihe L 363:368-408

Döhler H, Dämmgen U, Eurich-Menden B, Osterburg B, Lüttich M, Berg W, Bergschmidt A, Brunsch R (2002) Anpassung der deutschen Methodik zur rechnerischen Emissionsermittlung an internationale Richtlinien sowie Erfassung und Prognose der Ammoniak-Emissionen der deutschen Landwirtschaft und Szenarien zu deren Minderung bis zum Jahre 2010. Abschlussbericht, UBAVorhaben FKZ 29942245/02

Ellwanger G, Balzer S, Isselbächer T, Raths U, Schröder E, Ssymank A, Vischer-Leopold M, Zimmermann M (2008) Der nationale Bericht 2007 nach Art. 17 FFH-Richtlinie: Ein Überblick über die Ergebnisse unter besonderer Berücksichtigung der Käfer. Natursch Landschaftsplng 40(1):5-8

Eionet: http://biodiversity.eionet.europa.eu/article17 (4.8.2008)

European Commission, DG Umwelt (2007) Interpretation Manual of European Union Habitats. Version EUR 27, Juli. Brüssel. http:// ec.europa.eu/environment/nature/legislation/habitatsdirective/ docs/2007 07 im.pdf (4.8.2008)

FFH-Richtlinie art. 10 (http://www.bfn.de/0316_kohaerenz.html (4.8.2008)

Gauger T, Anshelm F, Schuster H, Erisman JW, Vermeulen AT, Draaijers GPJ, Bleeker A, Nagel H-D (2002) Mapping of ecosystem specific long-term trends in deposition loads and concentrations of air pollutants in Germany and their comparison with Critical Loads and Critical Levels. Final Report on behalf of Federal Environmental Agency (Umweltbundesamt), Berlin. BMU/UBA FE-No 29942 210. Part 1: Deposition Loads 1990-1999 Part 2: Mapping Critical Levels Exceedances. Institut für Navigation der
Universität Stuttgart. http://www.nav.uni-stuttgart.de/navigation/ forschung/critical_loads/ (4.8.2008)

ICP Modelling and Mapping (2004) Manual on Methodologies and Criteria for Mapping Critical Levels/Loads and geographical areas where they are exceeded. UBA-Texte 52/04, http://icpmapping.org/cms/zeigeBereich/11/manual_english.html (4.8.2008)

Nationaler Bericht (2007) Nationaler Bericht nach Art. 17 der FaunaFlora-Habitat-Richtlinie (92/43/EWG). http://www.bfn.de/0316 bericht2007.html (4.8.2008)

Öko-Data (2000) Bestimmung und Kartierung der Critical Loads \& Levels für Deutschland. UBA-Projekt FKZ 29773 011, Strausberg. http://www.umweltbundesamt.de/umweltbeobachtung/uid/ mapping/stickstoff.htm (4.8.2008)

Petersen B, Ellwanger G, Biewald G, Hauke U, Ludwig G, Pretscher P, Schröder E, Ssymank A (Bearb) (2003) Das europäische Schutzgebietssystem Natura 2000: Ökologie und Verbreitung von Arten der FFH-Richtlinie in Deutschland, Bd 1. Pflanzen und Wirbellose. Schriftenr Landschaftspfl Natursch 69/1

Petersen B, Ellwanger G, Bless R, Boye P, Schröder E, Ssymank A (Bearb) (2004) Das europäische Schutzgebietssystem Natura 2000: Ökologie und Verbreitung von Arten der FFH-Richtlinie in Deutschland, Bd 2. Wirbeltiere. Schriftenr Landschaftspfl Natursch 69/2

Pressemitteilung vom 16.5.2008, http://idw-online.de/pages/de/ news260637 (4.8.2008)

Raths U, Balzer S, Ersfeld M, Euler U (2006) Deutsche Natura-2000Gebiete in Zahlen. Natur und Landschaft 81(2):68-80

Rennwald E (2000) Bearb. Verzeichnis und Rote Liste der Pflanzengesellschaften Deutschlands. Schriftenr Vegetationskunde 35

Riecken U, Finck P, Raths U, Schröder E, Ssymank A (2006) Rote Liste der gefährdeten Biotoptypen Deutschlands. Zweite fortgeschriebene Fassung. Natursch Biol Vielf 34

Schiemenz H, Günther R (1994) Verbreitungsatlas der Amphibien und Reptilien Ostdeutschlands (Gebiet der ehemaligen DDR). Natur \& Text, Rangsdorf

Ssymank A, Hauke U, Rückriem C, Schröder E, Messer D (1998) Das europäische Schutzgebietssystem NATURA 2000. BfN-Handbuch zur Umsetzung der Fauna-Flora-Habitat-Richtlinie (92/43/ EWG) und der Vogelschutz-Richtlinie (79/409/EWG). Schriftenr Landschaftspfl Natursch 53 